\title{
A UNIFORM APPROXIMATION TO FOURIER COEFFICIENTS
}

\author{
M. L. JUNCOSA AND D. M. YOUNG
}

Let $f(x)$ be a real-valued function which is continuous in the interval $I:-1 \leqq x \leqq 1$ except at $N$ points at which it may have a finite jump. The sequence of its ordinary complex Fourier coefficients is given by

$$
a_{n}=\frac{1}{2} \int_{-1}^{1} f(x) e^{-i n \pi x} d x, \quad n=0, \pm 1, \cdots .
$$

It is the purpose of this note to show that the elements of the sequence of Riemann sum approximations to $a_{n}$,

$$
b_{n}(M)=\left\{\begin{array}{lr}
\frac{1}{2 M} \sum_{j=-M+1}^{M} f\left(\frac{j}{M}\right) e^{-i n \pi j / M}, & n=0, \pm 1, \cdots, \pm M, \\
0, & |n|>M
\end{array}\right.
$$

tend to $a_{n}$ uniformly as $M \rightarrow \infty$.

Let

$$
s_{m}=\sum_{j=-m}^{m} a_{j} e^{i j \pi x}, \quad m=0,1, \cdots
$$

and

$$
\sigma_{k}(x)=\frac{1}{k+1} \sum_{m=0}^{k} s_{m}(x), \quad k=0,1, \cdots
$$

Then

$$
\sigma_{k}(x)=\sum_{n=-k}^{k} A_{k, n} e^{i n \pi x}
$$

where

$$
A_{k, n}= \begin{cases}(1-|n| / k+1) a_{n}, & n=0, \pm 1, \cdots, \pm k \\ 0, & |n|>k .\end{cases}
$$

Let us cover the set of points of discontinuity of $f(x)$ in $I$ by a set $E$ which is the sum of a finite number of open intervals of lengths $\eta_{i}, i=1,2, \cdots, N$, each of which covers at most one point of discontinuity and such that $\sum_{i=1}^{N} \eta_{i} \equiv \eta<\epsilon / 6 F$, where $F=\mathrm{LUB}|f(x)|$ in

Received by the editors June 16, 1952. 
$I$. Then, on $I-I E$, by a generalization of Fejér's theorem on summability $(C, 1)$ of Fourier series (see, e.g., $\left[1\right.$, p. 66]) $\sigma_{k}(x)$ converges uniformly to $f(x)$. Therefore, let $M_{1}$ be such that $\left|\sigma_{k}(x)-f(x)\right|$ $<\epsilon / 6$ uniformly on $I-I E$ for $k \geqq M_{1}$. Henceforth, let $M$ $\geqq \max \left\{M_{1}, 3 N F / \epsilon\right\}$. Since, in general, $\left|\sigma_{k}(x)\right| \leqq F$ (see [1, p. 58]), on $I E$ we have $\left|\sigma_{k}(x)-f(x)\right| \leqq 2 F$.

For $|n| \leqq M$, using the above inequalities, we obtain

$$
\begin{aligned}
\left|b_{n}(M)-A_{M, n}\right| & =\frac{1}{2 M}\left|\sum_{j=-M+1}^{M}\left[f\left(\frac{j}{M}\right)-\sigma_{M}\left(\frac{j}{M}\right)\right] e^{-i n \pi j / M}\right| \\
& \leqq \frac{1}{2 M} \sum_{j=-M+1}^{M}\left|f\left(\frac{j}{M}\right)-\sigma_{M}\left(\frac{j}{M}\right)\right| \\
& \leqq \frac{1}{2 M}\left[\frac{2 M \epsilon}{6}+\sum_{i=1}^{N}\left(M \eta_{i}+1\right) 2 F\right]<\frac{2 \epsilon}{3} .
\end{aligned}
$$

For $|n|>M,\left|b_{n}(M)-A_{M, n}\right|=0$. For all $n$, we have

$$
\begin{aligned}
\left|A_{M, n}-a_{n}\right| & =\frac{1}{2}\left|\int_{-1}^{1}\left[\sigma_{M}(x)-f(x)\right] e^{-i n \pi x} d x\right| \\
& <\frac{1}{2}\left(\frac{\epsilon}{3}+2 \eta F\right)<\frac{\epsilon}{3} .
\end{aligned}
$$

Putting these results in the triangle inequality, we get $\left|b_{n}(M)-a_{n}\right|$ $<\epsilon$ uniformly for all integral $n$.

\section{REFERENCE}

1. Dunham Jackson, The theory of approximation, Amer. Math. Soc. Colloquium Publications, vol. 11, 1930.

Ballistic Research laboratories, Aberdeen Proving Ground, and UNIVERSITY OF MARYLAND 